\title{
Anatomical and radiological evaluation of the variation in the branching patterns and morphology of the aortic arch in the irish population- $A$ morphometric study
}

\author{
Katherine O Boyle ${ }^{1}$, Abdul-Kadhum Al-Modhefer ${ }^{1}$, Mark Worthington ${ }^{2}$
}

Boyle OK. Anatomical and radiological evaluation of the variation in the branching patterns and morphology of the aortic arch in the irish population- A morphometric study. Int J Anat Var. 2019;12(4): 40-45.

Introduction: Anatomical variations of the aortic arch are clinically significant for diagnostic and surgical procedures in the mediastinum. Our objective was to determine the arch morphology and incidence of variant branching patterns in both a cadaveric and patient population.

Materials and Methods: 194 CT scans were retrospectively analysed for branching variations and arch type. 18 cadavers were dissected with variations noted.

Results: Analysis of scans revealed normal branching in $77.32 \%$, bovine arch pattern in $14.43 \%$, direct origin of the left vertebral artery in $7.73 \%$ and a bovine arch with direct origin of the left vertebral artery in $0.52 \%$. A significant correlation $(p<0.0001)$ was found between arch type and age, with the mean age of a type 1 arch 51.1 years, type 2 arch 60.7 years, and type 3 arch 70.7 years. Direct origin of the left vertebral artery was found in one cadaver.

Conclusion: Correlation between arch type and age strengthens the concept of age-related unfolding of the aortic arch. A detailed understanding of the branching variations of the arch is important in the disciplines of anatomy, radiology and cardiothoracic surgery.

Key Words: Aortic arch; Anatomical variation; Morphometry; Radiology; Computed tomography

\section{INTRODUCTION}

$\mathrm{T}$ he standard configuration of the aortic arch develops as a result of a complex embryological process during the first few weeks of gestation. It begins with a primitive dorsal and ventral aorta connected by a number of embryological arches, each of which gives rise to a specific branch component. Three main branches normally stem from the arch in succession from right to left: the brachiocephalic trunk (which bifurcates into the right sub-clavian artery and the right common carotid artery), the left common carotid artery and the left subclavian artery, which serve to supply the head, neck and upper limbs. Abnormal persistence or regression of the embryologica arches may cause variations in the size, number and origin of these vessels. Variations in arch typology and the development of variant branches are reported in the literature with variations existing in $30 \%$ of individuals [1]. Usually these are asymptomatic however some variations may cause alterations in cerebral haemodynamics leading to aneurysm formation and vessel dissection, as well as producing compressive symptoms in a minority of individuals. Furthermore, in the expanding era of endovascular surgery and with the success of carotid artery stenting, an awareness of arch morphology is becoming increasingly important for interventional radiologists. Preoperative detection of these anomalies is crucial for the planning of safe surgical and radiological intervention and preventing potentially fatal ischaemic complications. Modern radiological modalities such as CT angiography provide clinicians with the tools to study the detailed vascular anatomy in clinical practice, however this anatomical 3D understanding is also supported with findings from cadaveric dissection. The objective of the present study was to evaluate the incidence of branching anomalies and arch characteristics in both a cadaveric and patient population. Through the use of dissection and computed tomography, clinically relevant morphometric data was gathered which can be applied to both the diagnostic and surgical field.

\section{MATERIALS AND METHODS}

Eighteen cadavers, including 10 female (mean age at death 93.9 years) and 8 male (mean age of death 83.9 years) were obtained from the Centre for Biomedical Sciences, Queen's University Belfast. The cadavers were predissected by medical students and the mediastinum was exposed to reveal the aorta and its branches. Each cadaver was assessed for the presence of variations pertaining to the arch. Photographs were documented of both the normal arch anatomy and of any variation present using a Nikon D3200 digital camera.

Two hundred and six anonymised consecutive Computed Tomography (CT) scans were obtained from the Department of Radiology at Royal Victoria Hospital. Consecutive patients undergoing diagnostic evaluation during a two year period from January 2014 to February 2016 were selected. Eleven of the 206 images were excluded due to technical limitations and poor image clarity; excessive thoracic kyphosis in one elderly female made measurement impossible. The final study population consisted of 194 patients (125 males, 69 females; mean age $62( \pm 17.36)$ years; age range $18-96$ years). The patients were assigned to 7 age categories; $18-30(n=13,6.7 \%), 31-40(n=14$ $7.22 \%), 41-50(n=14,7.22 \%), 51-60 \quad(n=33,17.01 \%), 61-70(n=50,25.77 \%)$ $71-80(\mathrm{n}=48,24.74 \%)$ and $81-96(\mathrm{n}=22,11.34 \%)$. Ethical approval was obtained from Queens University Belfast ethics board. Informed consent

${ }^{1}$ Department of Medicine, Dentistry and Biomedical Sciences, Queen's University Belfast, Whitla Medical Building, Belfast, County Antrim, Northern Ireland. BT7 1NN, UK; ${ }^{2}$ Department of Radiology, Royal Victoria Hospital, Belfast, Northern Ireland, UK

Correspondence: Dr. Katherine O Boyle, Department of Medicine, Dentistry and Biomedical Sciences, Queen's University Belfast, Whitla Medical Building, Belfast, County Antrim, Northern Ireland. BT7 1NN, UK. Telephone+447518554467; E-mail: koboyle07@qub.ac.uk org/licenses/by-nc/4.0/), which permits reuse, distribution and reproduction of the article, provided that the original work is properly cited and the reuse is restricted to noncommercial purposes. For commercial reuse, contact reprints@pulsus.com 
from the patients was not required. All exams had previously been reported by Consultant Radiologists. The patient reports or personal demographic details were not accessed during review of imaging.

All CT scans were performed using 64-slice CT scanners (CT1 Phillips Ingenuity 128 and CT2 Phillips ICT 128). Imaging parameters utilised 50 $\mathrm{cm}$ and $45.6 \mathrm{~cm}$ Field of View (FOV) respectively and slice thickness of 2 $\mathrm{mm}$ with $50 \%$ overlap, slice increment of $1 \mathrm{~mm}$ and slice collimation of $64 \times 0.625$. A solution of iodinated contrast was administered intravenously (300 $\mathrm{mg} \mathrm{I} / \mathrm{ml}$ ), with a volume acquisition in the arterial phase. The images were reviewed on a PACS workstation using Multiplanar Reconstructions (MPR). Images were analysed in axial, coronal and sagittal planes with measurements taken in the parasagittal plane.

The scans were evaluated by assessing variations in the anatomy and branching patterns of the arch. The arch was categorised into three types based on the vertical distance from the proximal wall of the origin of the brachiocephalic trunk to the highest point of the aortic arch (Figure 1). The ratio of this distance to the diameter of the left common carotid artery (Figure 2) is $<1$ in a type 1 arch, between $1-2$ in a type 2 arch and $>2$ in a type 3 arch [2]. The diameters of the brachiocephalic trunk, right subclavian artery and the arch of the aorta at its highest point were measured in 104 scans. Where present, the diameter of the left vertebral artery arising from the arch was also measured.

\section{Statistical analysis}

All statistical analyses were performed using SAS 9.3 [3]. Summary statistics and frequency tables were generated to describe the following characteristics about the sample population: age, gender, variations, vertical distance, diameters of the left common carotid artery, right subclavian artery, brachiocephalic trunk and arch. Data were checked for normality and homogeneity of variance by histograms, QQ-plots, and formal statistical tests as part of the PROC UNIVARIATE procedure of SAS. Non-statistically significant interactions were sequentially removed from the model. Data are presented as the mean with Standard Deviation (SD). Differences in the ages of the patients, represented as seven age categories (18-30; 31-40; $41-50 ; 51-60 ; 61-70 ; 71-80 ; 81-96)$ among the three branching types were analysed using PROC GLM one-way ANOVA followed by the Tukey test (SAS 9.3). P values less than 0.05 were considered statistically significant. The correlations between age, gender, type of arch, variations, diameter of vessels and diameter of the arch were statistically analysed using Pearson correlation procedure in SAS.

\section{RESULTS}

Of the 18 cadavers one branching variation was found (5.6\%) where the left vertebral artery arose directly from the arch between the left common carotid artery and left subclavian artery in a 97 year old male (Figure 3). The branches of the aortic arch from right to left were the brachiocephalic trunk, left common carotid artery, left vertebral artery and left subclavian artery. No additional branching variations of the thoracic aorta were noted. The remaining 17 cadavers $(94.4 \%)$ had a normal left-sided arch with threebranch configuration (Figure 4).

Of the 194 CT scans, arch type was determined for each patient based on the anatomical illustrations depicted in (Figure 5) and methodology of a previous study by Demertzis et al. [2]. Fifty-five patients (28.4\%) had a type 1 arch, 59 patients (30.4\%) had a type 2 arch and the type 3 arch was the

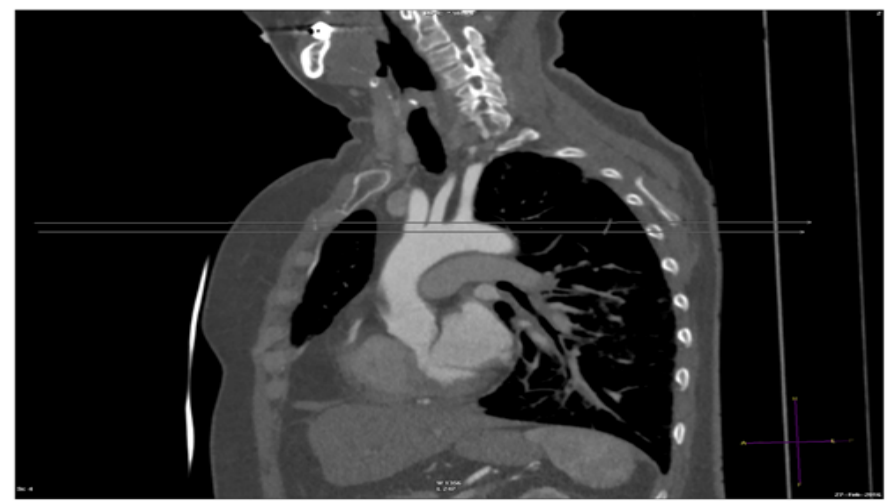

Figure 1) Measurement technique-Vertical distance taken between the horizontal planes passing through the top of the arch and through the origin of the brachiocephalic trunk in the parasagittal plane.

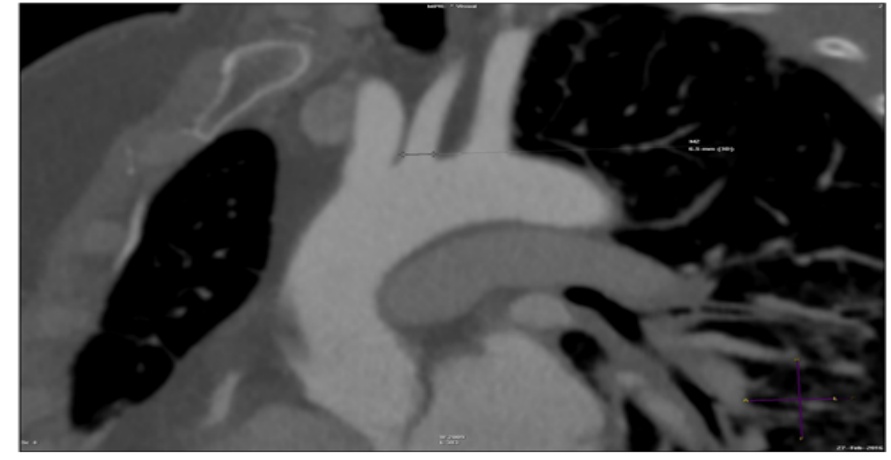

Figure 2) Measurement technique-Diameter of the left common carotid artery taken in the parasagittal plane.

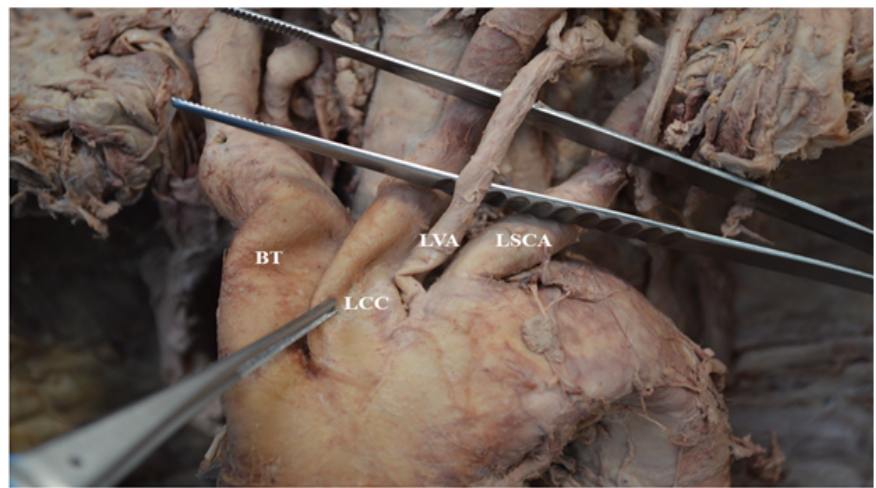

Figure 3) Photograph of left vertebral artery arising directly from the arch in a 97 year old male cadaver (BT-Brachiocephalic trunk, LCC- Left common carotid artery, LVA- Left vertebral artery, LSCA- Left subclavian artery).

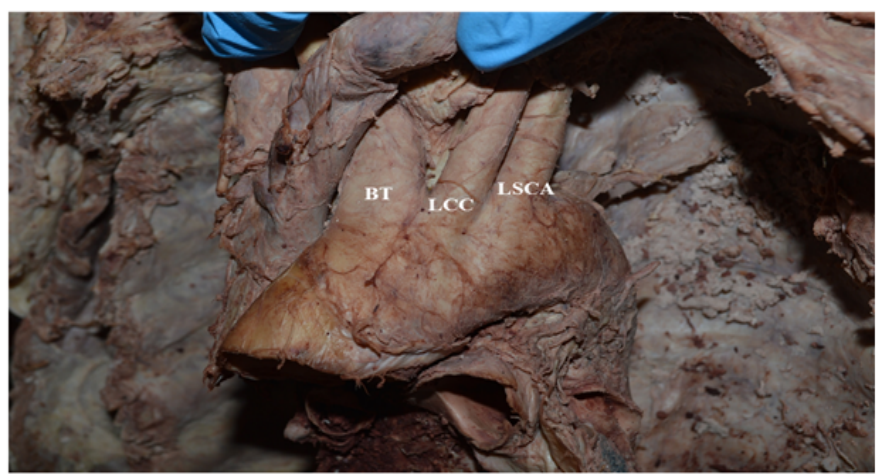

Figure 4) Photograph of normal aortic arch branching (BT- Brachiocephalic trunk, LCC- Left common carotid artery, LSCA- Left subclavian artery).

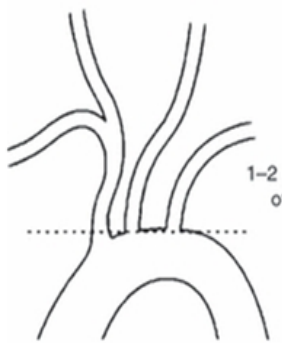

Type I

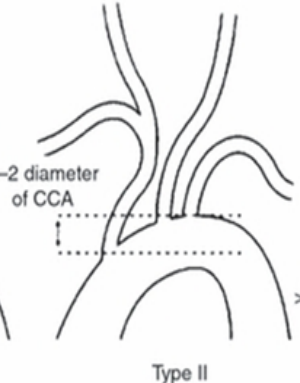

Type II

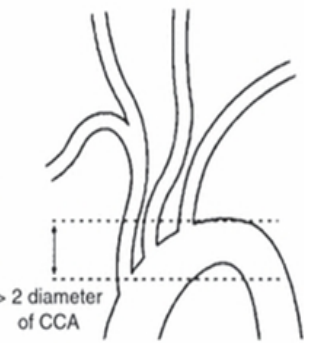

Type III
Figure 5) Illustration of arch types 1-3, adapted from Demertzis et al. (2010) (CCALeft Common Carotid Artery).

most commonly observed, prevalent in 80 patients (41.2\%). The grouping of patients into the seven age categories; 18-30, 31-40, 41-50, 51-60, 61-70, 71-80 and 81-96 showed age related trends with arch type (Table 1). Pearson correlation coefficient $(r)$ demonstrated a statistically significant association 
$(\mathrm{r}=0.47 ; \mathrm{p}<0.0001)$ between age and arch type and between vertical distance and arch type $(\mathrm{r}=0.79 ; \mathrm{p}<0.0001)$, with a type 3 arch being more prevalent in the older age categories. There was a significant correlation between age and the vertical distance $(\mathrm{r}=0.53 ; \mathrm{p}<0.0001)$, as measured from the top of the arch to the origin of the brachiocephalic trunk. Age was however not significantly associated with the diameters of the vessels or the arch. Gender was not significantly associated with any of the measured variables, which are displayed in (Table 2).

All of the 194 arches were left-sided, 150 (77.32\%) showing the usual three branch configuration of brachiocephalic trunk, left common carotid artery and left subclavian artery. A bovine arch, the common origin of the brachiocephalic trunk and left common carotid artery, was present in 28 $(14.43 \%)$ patients and the left vertebral artery arose directly from the arch in $15(7.73 \%)$ cases. An interesting case of both a bovine arch and direct origin of the left vertebral artery was also discovered in a 59 year old female. These findings together with gender breakdown are shown in (Table 3).

\section{DISCUSSION}

To the best of our knowledge, this is the first Irish study to evaluate the incidence of branching anomalies and arch characteristics in both a cadaveric and patient population, through the use of dissection and computed tomography. Previous studies in the literature have reported discrepancies in variations of the morphology and branching configuration of the aortic arch, which occur frequently as a result of embryological dysmorphism or age-related degenerative change.

The aortic arch can be morphologically classified into three types according to the relationship between the brachiocephalic trunk and the arch (Figure 5).
In a type 1 arch the supra-aortic branches arise from a horizontal plane across the outer curvature of the arch. The vertical distance, from the proximal origin of the brachiocephalic trunk to the top of the arch is less than 1 of the left common carotid artery diameter. In a type 2 arch the brachiocephalic trunk arises between the horizontal planes through the inner and outer curvatures. The vertical distance is between 1 and 2 times the diameter of the left common carotid artery. In a type 3 arch the brachiocephalic trunk will originate inferior to the horizontal plane through the inferior curvature of the arch, and the vertical distance will be greater than 2 times the diameter of the left common carotid artery [4].

The present study found a significant correlation (Pearson $(r=0.47 ; p<0.0001)$ between arch type and age, with the type 3 arch occurring more frequently in the older age categories. The mean age (SD) of a type $1 \mathrm{arch}$ is $51.1(17.42)$ years increasing to 60.7 (15.89) years with a type 2 arch and 70.7 (13.52) years with a type $3 \mathrm{arch}$. These findings are in broad agreement with a recent Japanese study by Kojima et al. as shown in (Table 4) [5]. However, Demertzis et al. performed a similar study and their occurrence of type 3 arches was much lower at $17 \%$ compared to $41.2 \%$ (present study) and $64.0 \%$ (Kojima et al.) $[2,5]$. Their relatively small sample size and narrower age range were likely limiting factors however it is unclear why there are comparatively fewer type 3 arches given its older age profile. The larger population size and wider age range of the present study should lead to more reliable results. A detailed breakdown of arch type according to age is shown in (Table 1).

The Type 3 arch tends to occur in older individuals as the aorta elongates and unfolds as a consequence of ageing and long-standing hypertension [6]. The elongation of the ascending aorta and arch causes an inferior displacement of the origins of the aortic branches and the resultant increased angulation

\section{TABLE 1}

Arch type according to age category.

\begin{tabular}{cccccccccc}
\hline Arch & $18-30$ & $31-40$ & $41-50$ & $51-60$ & $61-70$ & $71-80$ & $81-96$ & $\begin{array}{c}\text { Number of } \\
\text { patients }\end{array}$ & \begin{tabular}{c} 
Mean age (SD) years \\
\hline Type 1
\end{tabular} \\
\hline Type 2 & 4 & 10 & 5 & 7 & 17 & 8 & 0 & $55(28.4 \%)$ & $51.1(17.42)$ \\
\hline Type 3 & 1 & 2 & 6 & 15 & 15 & 15 & 2 & $59(30.4 \%)$ & $60.7(15.89)$ \\
\hline
\end{tabular}

TABLE 2

Summary of results of radiological measurements (LCC: Left Common Carotid Artery; RSC: Right Subclavian Artery; BT: Brachiocephalic Trunk)

\begin{tabular}{cccccccc}
\hline Variable & Mean & Minimum & Maximum & SD & Number missing & Range & Coefficient of variation \\
\hline Age (Years) & 62.10 & 18.00 & 96.00 & 17.36 & 0 & 78.00 & 27.95 \\
\hline Vertical distance $(\mathrm{cm})$ & 1.39 & 0.20 & 5.20 & 0.99 & 0 & 5.00 & 71.17 \\
\hline Diameter RSC $(\mathrm{cm})$ & 0.71 & 0.39 & 1.05 & 0.12 & 0 & 0.66 & 17.47 \\
\hline Diameter RCC $(\mathrm{cm})$ & 1.06 & 0.65 & 1.52 & 0.19 & 89 & 0.87 & 18.20 \\
\hline Diameter Arch $(\mathrm{cm})$ & 2.38 & 1.67 & 3.12 & 0.13 & 89 & 1.45 & 12.81 \\
\hline Diameter BT $(\mathrm{cm})$ & 1.20 & 0.72 & 1.86 & 0.21 & 89 & 17.26 \\
\hline
\end{tabular}

TABLE 3

Incidence of variation of arotic branching in patient population.

\begin{tabular}{ccccc}
\hline Variation & Male & Female & Total & Total $\%$ \\
\hline Normal & $93(62 \%)$ & $57(38 \%)$ & 150 & $77.32 \%$ \\
\hline Bovine & $21(75 \%)$ & $7(25 \%)$ & 28 & $14.43 \%$ \\
\hline Left Vertebral (LVA) & $11(73.3 \%)$ & $4(26.7 \%)$ & 15 & $7.73 \%$ \\
\hline Bovine and LVA & $0(0 \%)$ & $1(0.61 \%)$ & 1 & $0.52 \%$ \\
\hline Right aberrant subclavian & $0(0 \%)$ & $0(0 \%)$ & 0 & $0 \%$ \\
\hline
\end{tabular}

\section{TABLE 4}

Breakdown of arch by patients (mean age (SD) in years) in present and previous radiological studies.

\begin{tabular}{ccccccccc}
\hline Type of Arch age (SD) & Patients (n) & Mean & Type 1 age (SD) & Mean & Type 2 & Mean age (SD) & Type 3 (SD) & Mean age \\
\hline Present study (17.36) & 194 & 62.1 & $55(28.4 \%)$ & $51.1(17.42)$ & $59(30.4 \%)$ & $60.7(15.89)$ & $80(41.2 \%)$ & $70.7(13.52)$ \\
\hline Kojima et al. (2016) (12.7) & 139 & 68.0 & $20(14.4 \%)$ & $56.1(18.0)$ & $30(21.6 \%)$ & $66.3(11.5)$ & $89(64.0 \%)$ & $71.7(9.8)$ \\
\hline Demertzis et al. (2010) (9.9) & 92 & 69.4 & $43(47 \%)$ & $67.35(8.66)$ & $33(36 \%)$ & $68.67(11.57)$ & $16(17 \%)$ & $74.67(7.52)$ \\
\hline
\end{tabular}


makes it more difficult to traverse the arch and access the carotid artery [7]. The steep arch is hard to manoeuvre and during cerebral aneurysm catheterisation, emboli dislodgment may occur due to manipulation within a highly stenotic vessel [8]. The common carotid artery is usually accessed via the femoral artery approach, however in cases of a complex arch the brachial or radial artery may be preferred [9].

The aortic arches in both cadaveric and patient populations were analysed for branching variations, which occur in around 30\% of individuals as a result of deviation from normal arch development [10]. Awareness of the embryological process is key to understanding the formation of arch anomalies.

The $3^{\text {rd }}$ week of gestation marks the beginning of aortic development, with a primitive dorsal and ventral aorta, linked together by six pairs of aortic arches, from which the adult aorta and its branches are derived. The first, second and fifth arches regress. The $3^{\text {rd }}$ arch gives rise to the common and internal carotid arteries, the $4^{\text {th }}$ right arch forms part of the right subclavian artery whereas the $4^{\text {th }}$ left arch forms the segment of arch between the left common carotid and left subclavian arteries. In addition to the arches, the two ventral aortae fuse to become the aortic sac, from which arises the ascending aorta and brachiocephalic trunk. The two dorsal aortae also fuse to form the descending aorta and additionally they give off intersegmental arteries. The left subclavian artery arises from the left $7^{\text {th }}$ intersegmental artery entirely. The proximal aspect of the right subclavian artery is derived from the right $4^{\text {th }}$ aortic arch, whereas the distal aspect originates from the right dorsal aorta and the right $7^{\text {th }}$ intersegmental arteries [11]. Any disruption to this developmental process will produce a variant branching pattern [12].

In the present study of 194 patients, variations were found in $22.68 \%$ of patients. A bovine arch was present in $14.43 \%$ while $7.73 \%$ showed a direct origin of the left vertebral artery from the arch. Our results were closely correlated with those of Patil et al. who reported the normal branching pattern in $77.3 \%$, bovine arch in $14.6 \%$ and direct origin of the left vertebral artery in $8 \%[13]$.

The normal configuration of the aortic arch consists of the brachiocephalic trunk, left common carotid artery and left subclavian artery in right to left succession (Figures 6 and 7). This occurs in around $70 \%$ of individuals, ranging between $64.9-94.3 \%$ in the literature [14]. Normal branching was evident in $77.32 \%$ of patients in this study.

The brachiocephalic trunk may develop a common origin with the left common carotid artery, in what is known as a bovine arch (Figure 8). This is the most common variation, present in around $13 \%$ of individuals ranging between 10 and $27 \%[15,16]$. Our result of $14.43 \%$ is in agreement with the above two studies and also with Acar et al. who reported an incidence of $14.91 \%$ [17]. Although asymptomatic, if this common trunk is occluded, critical ischaemic consequences can occur as both carotid arteries arise from this single trunk.

The vertebral artery normally originates from the first part of the subclavian artery and enters the foramen tranversarium of the $6^{\text {th }}$ cervical vertebra before ascending to provide blood supply to the posterior circulation [18]. The prevertebral part of the left vertebral artery develops from the proximal part of the dorsal $7^{\text {th }}$ intersegmental artery. This is the only intersegmental artery to persist, forming the left subclavian artery. However if there is persistence of the $6^{\text {th }}$ intersegmental artery, the vessel will arise from the arch between

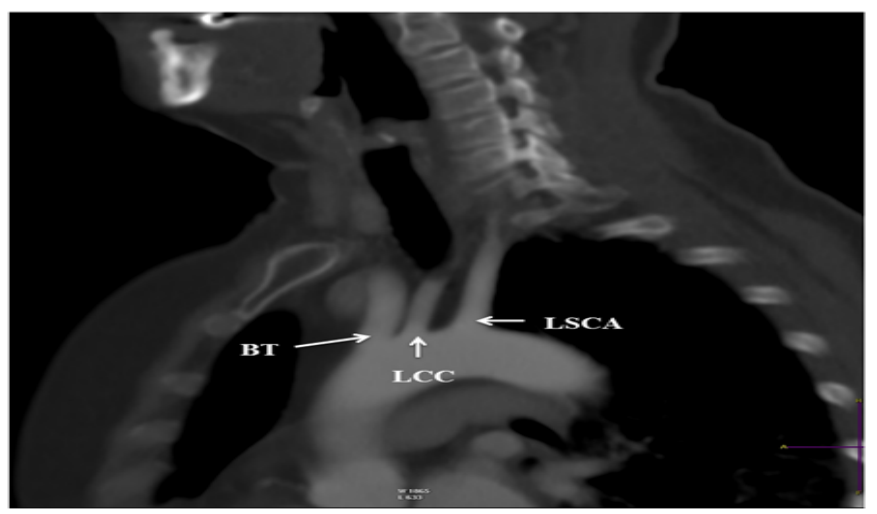

Figure 6) CT image of normal aortic arch branching (BT-Brachiocephalic trunk, LCC-Left common carotid artery, LSCA-Left subclavian artery).

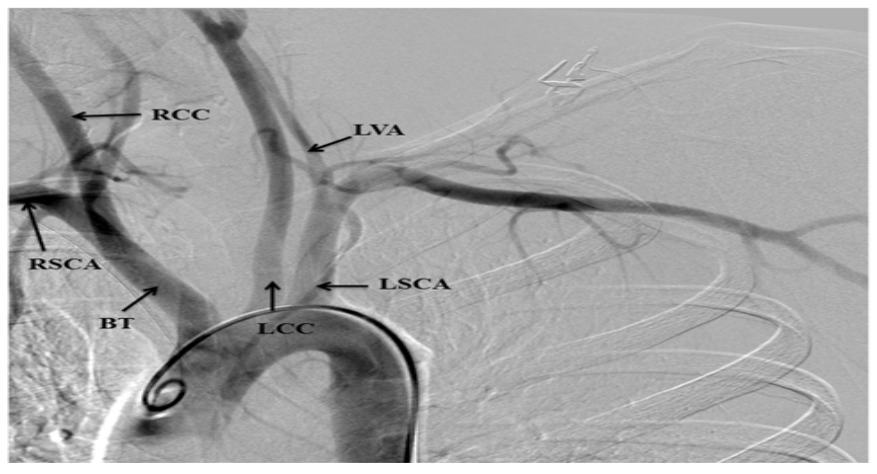

Figure 7) Catheter angiogram showing normal arch branching (BT- Brachiocephalic trunk, RSCA-Right subclavian artery, RCC-Right common carotid artery, LCC-Left common carotid artery, LVA- Left vertebral artery, LSCA- Left subclavian artery).

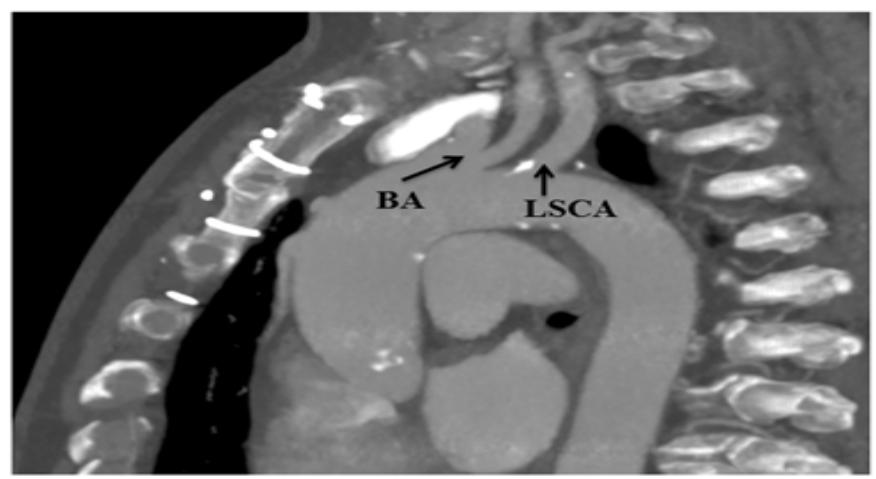

Figure 8) Bovine arch-Common origin of brachiocephalic trunk and left common carotid artery (BA- Bovine arch, LSCA- Left subclavian artery).

the left common carotid and left subclavian arteries. Less commonly, if there is persistence of the $8^{\text {th }}$ intersegmental artery, it will originate distal to the left subclavian artery. Persistence of the $4^{\text {th }}$ and $5^{\text {th }}$ intersegmental arteries will cause duplication of the vertebral artery [19]. The vertebral part, extending between foramen transversarium C1-C6, develops from postcostal longitudinal anastomoses between the $1^{\text {st }} 7^{\text {th }}$ intersegmental arteries $[10,20]$. The origin of the left vertebral artery from the arch is the second most common aortic arch variation, with an incidence around $2.4-5.8 \%$ and is increased by $40 \%$ in Down's syndrome [19,21]. Other variant origins of the vertebral artery include the common carotid artery or its branches which are important to recognise prior to surgical ligation of the common carotid artery, which would compromise blood supply of the posterior fossa [22]. The vertebral artery may be duplicated or it may possess a bifid origin from the arch which poses a greater risk of injury [23]. These variations are generally asymptomatic however symptoms can arise if there is disruption of cerebral haemodynamics, increasing susceptibility to intracranial aneurysms due to pulsatile and turbulent blood flow [24]. A variant origin and longer extra-cranial course of the left vertebral artery may also predispose to arterial dissection, as reported by Komiyama et al. who found a much higher incidence of vertebral artery dissection in those with direct origin of the left vertebral artery from the arch compared to those arising from the subclavian artery [25].

Due to its small size, pre-operative detection of a variant left vertebral artery can often be missed [26]. If the diameter is $<3.5 \mathrm{~mm}$ it is considered hypoplastic and is often obscured by other supra-aortic branches [27]. Hypoplastic vessels are at a greater risk of iatrogenic injury and subsequent vertebro-basilar insufficiency [20]. The average diameter of the left vertebral artery from the arch in this study was $3 \mathrm{~mm}$. In the present study the left vertebral artery arose from the arch in 1 out of 18 cadavers, an incidence of $0.56 \%$, in line with Ughade et al. who found this variation in 1 out of 20 cadavers [10]. Out of the 194 CT scans, $7.73 \%$ of patients displayed the variant left vertebral artery arising from the arch (Figure 9). This closely correlates with the results from a study of 81 cadavers from a South Australian population, reporting an incidence of $7.41 \%$ [28]. Another cadaveric study of 25 Korean cadavers found the direct origin of the left vertebral artery in $8 \%$ of cases, and $8 \%$ of 75 Indian cadavers also exhibited this variation $[13,29]$. The highest incidence of direct origin of the left vertebral artery in 
the literature was $13.3 \%$ in a cadaveric and radiological study of Romanian individuals [30].

Although not observed in the present study, an aberrant right subclavian artery poses great importance for radiologists and surgeons with a reported prevalence [31,32]. The right subclavian artery usually develops from the right $4^{\text {th }}$ aortic arch, however involution of this arch and persistence of the $7^{\text {th }}$ intersegmental artery with the right dorsal aorta results in the origin of the right subclavian artery from the proximal descending aorta [33,34]. As the vessel loops backwards in a left to right direction, it may encircle the trachea and oesophagus producing a vascular ring anomaly. This abnormal development also produces a right non-recurrent laryngeal nerve no longer hooking under the right subclavian artery in the usual position. Instead it originates directly from the cervical aspect of the vagus nerve which may pose problems during thyroidectomy and tracheostomy [14,34,35]. Figure 10 displays an axial, sagittal and para-coronal view of a CT scan showing an aberrant right subclavian artery passing posterior to the oesophagus, which is the usual course in $80 \%$ of cases. It may also pass between the oesophagus and trachea in $15 \%$ of cases and anterior to the trachea in $5 \%$ of cases [36].
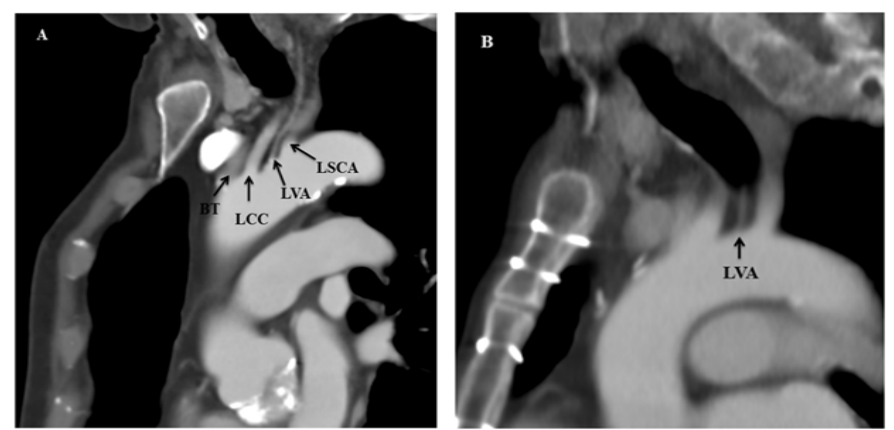

Figure 9) (A, B) Left vertebral artery arising directly from the arch (BTBrachiocephalic trunk, LCC- Left common carotid artery, LVA- Left vertebral artery, LSCA. Left subclavian artery).
Its relation to these structures can cause compressive symptoms and this anomaly is often referred to as "dysphagia lusoria" meaning a trick of nature [14].

All of the above variations were present in an angiographic study of 1359 arches, which is the largest radiological study evaluating aortic arch branching variations to date [16]. They reported $71 \%$ normal branching pattern, $22 \%$ bovine arch, $3 \%$ direct origin of the left vertebral artery and $0.5 \%$ aberrant right subclavian artery. The variant branching pattern and configuration of the aortic arch is highly significant in the advancing field of endovascular medicine. Atherosclerotic stenosis of the carotid arteries is common, and the prevalence is increasing with an ageing and obese population. Fortunately, successful management and subsequent stroke prevention can be achieved with catheterisation and stent placement, however navigation of the arch vessels has proved difficult in the presence of branching variations. Unfavourable anatomical characteristics particularly in the elderly population, as has been shown in the present study, render carotid artery stenting technically challenging. They increase the likelihood of technical failure and thrombo-embolic complications [8], and can be established with pre-interventional imaging, the gold standard being carotid angiography. Imaging of both carotid arteries can establish the severity of stenosis and the vascular morphology of the patient, enabling accurate planning and catheter selection $[6,7,14,16]$. Radiological measurements are subject to variations and limiting factors in this study include precision of distance measurement using the software tools, scan quality and calcification of vessels. CT angiography serves as a superior technique for evaluating aortic variations, however in this study these were not readily available.

\section{CONCLUSION}

Morphological information obtained from the study of the aortic arch is clinically relevant to interventional radiologists and vascular surgeons, particularly in the field of catheterisation and carotid stenting. Cadaveric study enables a 3D understanding of the configuration of the arch and its vessels that cannot be fully appreciated on clinical imaging. With advances in MR and CT angiography, detection of variant vessels and determination of arch characteristics can be made pre-operatively in patients undergoing
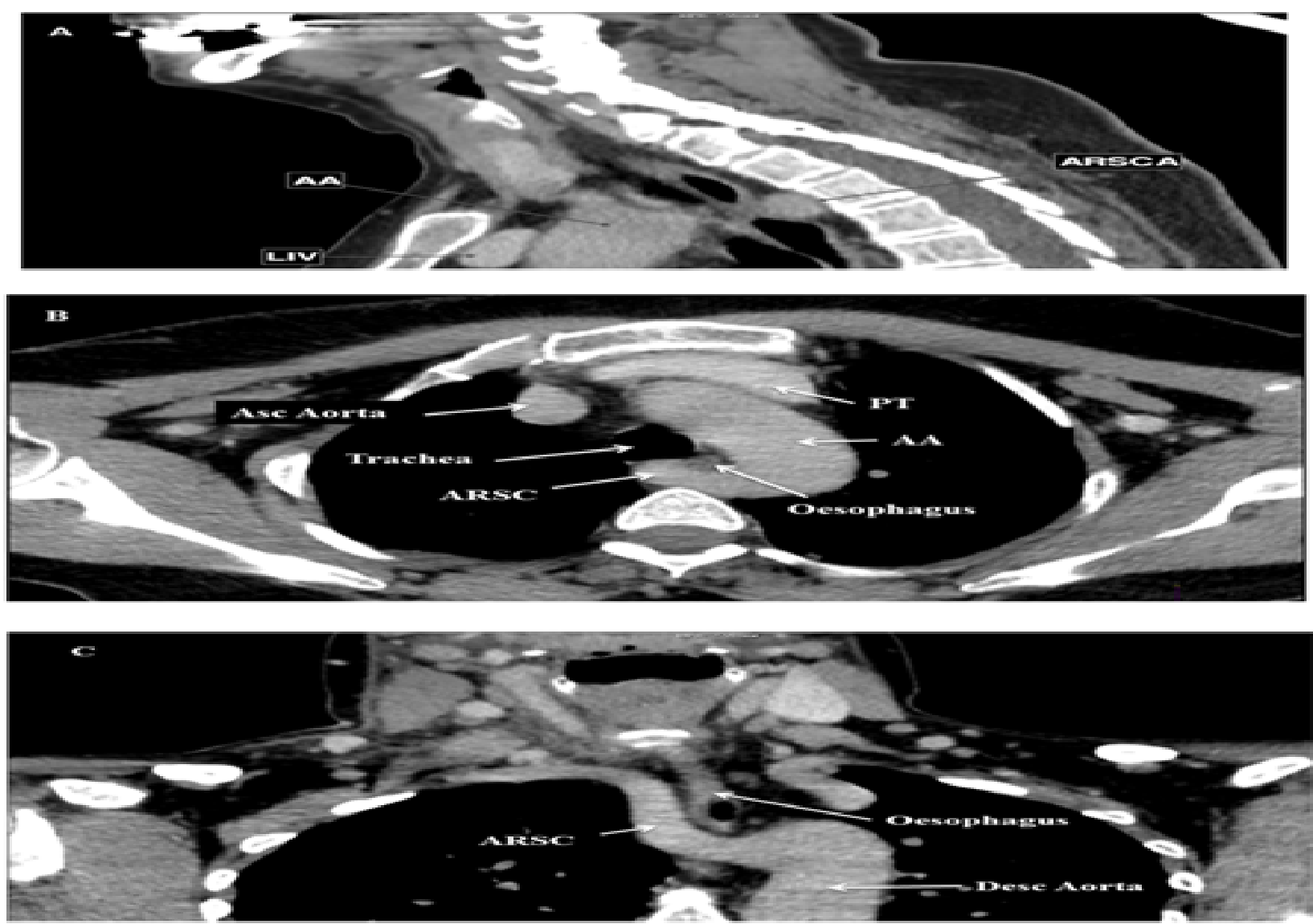

Figure 10) (A, B and C) Aberrant right subclavian artery (ARSC). A Sagittal view. B Axial view-ARSC passing posterior to oesophagus from left to right. C Para-coronal view with ARSC crossing the midline. (AA-Aortic arch, ARSC-Aberrant right subclavian artery, PT- Pulmonary trunk) 
head, neck and thoracic surgery. In this radiological study of 194 patients, the normal anatomy was observed in $77.32 \%$, a bovine arch in $14.43 \%$ and a direct origin of the left vertebral artery from the arch in $7.73 \%$ of cases. The direct origin of the left vertebral artery was found in $5.6 \%$ of 18 cadavers and considerable deviation in the origin of arch vessels from the mid-vertebral line was observed. These results are in keeping with existing literature. From the radiological analysis a statistically significant correlation was found between arch type and age in line with expected arch unfolding and elongation in elderly individuals. Complex arch anatomy is often evident in older individuals, leading to higher peri-operative complications of stroke, MI, and death following endovascular procedures such as carotid artery stenting. Variable aortic morphology including variant branching patterns and highly elongated arches contribute to the difficulty in performing interventional procedures in this region. The present study corroborates the findings of Kojima et al. revealing a significant link between ageing and the configuration of the aortic arch. Future studies should address correlations and potential causal links between variations and clinical morbidity, or vice versa. Pre-operative imaging with CT angiography is the gold standard in detecting branch variations and arch types. It enables surgical planning, correct instrument and catheter selection, as well as improved diagnostics and reduced complication rates. The present study establishes the frequency of variations in both cadaveric and patient populations and also confirms the correlation of arch typology with age.

\section{ACKNOWLEDGEMENTS}

The author would like to thank the cadaver donors and families for their contribution to this research.

\section{CONFLICTS OF INTEREST}

The author has no conflicts of interest to declare.y.

\section{REFERENCES}

1. Nanda NC. Comprehensive Textbook of Echocardiography. Delhi: Jaypee Brothers Medical Publishers Ltd. 2013;1-2044

2. Demertzis S, Hurni S, Stalder M, et al. Aortic arch morphometry in living humans. J Anat. 2010;217:588-96.

3. SAS Statistical Analysis software (SAS) version 9.3 (SAS Inst. Inc., Cary, NC, USA).

4. Madhwal S, Rajagopal V, Bhatt CT, et al. Predictors of difficult carotid stenting as determined by aortic arch angiography. J Invasive Cardiol. 2008;20:200-4.

5. Kojima A, Saga I. Effect of aging on the configurational change of the aortic arch. Geriatric Care. 2016;2.

6. Sugawara J, Hayashi K, Yokoi T, et al. Age-associated elongation of the ascending aorta in adults. JACC Cardiovasc Imag. 2008;1:739-48.

7. Bates E, Babb J, Casey DE, et al. A report of the American College of Cardiology Foundation Task Force on Clinical Expert Consensus Documents (ACCF/SCAI/SVMB/SIR/ASITN Clinical Expert Consensus Document Committee on Carotid Stenting. J Am Coll Cardiol. 2007;49:126-70.

8. Lam RC, Lin SC, DebRubertis B, et al. The impact of increasing age on anatomic factors affecting carotid angioplasty and stenting. J Vasc Surg. 2007; 45:875-80.

9. Dahm JB, Van Buuren F, Hansen C, et al. The concept of an anatomy related individual arterial access: lowering technical and clinical complications with transradial access in bovine- and type-III aortic arch carotid artery stenting. VASA. 2011;40:468-73.

10. Ughade JM, Kardile PB, Ughade MN, et al. Anomalous arch of aorta giving rise to left vertebral artery. Int J Biol Med Res. 2012;3:2452-4.

11. Kau T, Sinzig M, Gasser J, et al. Aortic development and anomalies. Semin Intervent Radiol. 2007;24:141-52.

12. Jalali KB, Asadi MH, Rahimian E, et al. Anatomical Variations in Aortic Arch Branching Pattern. Arch Iran Med. 2016;19:72-4.

13. Patil S, Meshram M, Kamdi N, et al. Study on branching pattern of aortic arch in Indian. Anat Cell Biol. 2012;45:203-6.

14. Natsis KI, Tsitouridis IA, Didagelos MV, et al. Anatomical variations in the branches of the human aortic arch in 633 angiographies: clinical significance and literature review. Surg Radiol Anat. 2009;31:319-23.

15. Layton KF, Kallmes DF, Cloft HJ, et al. Bovine Aortic Arch Variant in Humans: Clarification of a Common Misnomer. AJNR Am J Neuroradiol. 2006;27:1541-2.

16. Rea G, Valente T, Iaselli F, et al. Multi-detector computed tomography in the evaluation of variants and anomalies of aortic arch and its branching pattern. Ital J Anat Embryol. 2014;119:180-92.

17. Acar M, Ulusoy M, Zararsiz I, et al. Anatomical variations in the branching of human aortic arch. Biomed Res. 2003;24:531-5.

18. Yuan SM. Aberrant Origin of Vertebral Artery and its Clinical Implications. Braz J Cardiovasc Surg. 2016;31:52-9.

19. Jung S, Jung C, Bae YJ, et al. Duplicated Origin of the Left Vertebral Artery: A Case Report and Embryological Review. Neurointervention. 2016;11:50-4

20. Poonam S, Sharma T, Singla RK. Incidence of anomalous origins of vertebral artery-Anatomical study and clinical significance. J Clin Diagn Res. 2010;4:2626-31.

21. Rathore MH, Sreenivasan V. Vertebral and right subclavian artery abnormalities in the Down syndrome. Am J Cardiol. 1989;63:1528-9.

22.Satti SR, Cerniglia CA, Koenigsberg RA. Cervical Vertebral Artery Variations: An Anatomic Study. AJNR Am J Neuroradiol. 2007;28:97680.

23. Shhadeh A, Sair HI, Kanamalla US. Bifid direct aortic arch origin of left vertebral artery: a unique vascular anomalous. J Vasc Interv Radiol. 2007; 18:1051-3.

24. Tapia GP, Zhu X, Xu J, et al. Incidence of branching patterns variations of the arch in aortic dissection in Chinese patients. Medicine (Baltimore). 2015;94:795-802

25. Komiyama M, Morikawa T, Nakajima $\mathrm{H}$, et al. High incidence of arterial dissection associated with left vertebral artery of aortic origin. Neurol Med Chir 2001;41:8-11.

26.Suzuki K, Kazui T, Bashar AH, et al. Total aortic arch replacement in patients with arch vessel anomalies. Ann Thorac Surg. 2006;81:2079-83.

27. Junagade B, Mukherjee A. The morphometric study of the normal and anomalous branching pattern of the Aortic arch by cadaveric dissection. Int J Med Res Rev. 2015;3:461-9.

28. Bhatia K, Ghabriel MN, Henneberg M. Anatomical variations in the branches of the human aortic arch: a recent study of a South Australian population. Folia Morphol. 2005; 64:217-23.

29. Shin IY, Chung YG, Shin WH, et al. A morphometric study on cadaveric aortic arch and its major branches in 25 korean adults : the perspective of endovascular surgery. J Korean Neurosurg Soc. 2008;44:78-83.

30. Manole AM, Illiescu DM, Baz R, et al. Morphological characteristics of the aortic arch organization. ARS Medica Tomitana. 2013;19:67-73.

31. Karacan A, Türkvatan A, Karacan K. Anatomical variations of aortic arch branching: evaluation with computed tomographic angiography. Cardiol Young. 2014;24:485-93.

32. Nachtigan J, Rosen E. Arteria Lusoria and Truncus Bicaroticus: A Unique Aortic Arch Anatomic Variation. Vasc Dis Manag. 2013;10.

33. Nayak S, Pai M, Prabhu L, et al. Anatomical organisation of aortic arch variations in India: embryological basis and review. J Vasc Bras. 2006;5: 95-100.

34. Polguj M, Chrzanowski L, Kasprzak JD, et al. The aberrant right subclavian artery (arteria lusoria): the morphological and clinical aspects of one of the most important variations-a systematic study of 141 reports. Scientific World J. 2014;4:1-6.

35. Liechty J, Shields T, Anson B. Variations pertaining to the aortic arches and their branches; with comments on surgically important types. Q Bull Northwest Univ Med Sch. 1957;31:136-43.

36. Lale P, Toprak U, Yagiz G, et al. Variations in the Branching Pattern of the Aortic Arch Detected with Computerized Tomography Angiography. Adv Radiol. 2014;5:1-6. 